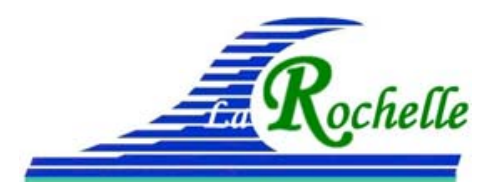

XV $V^{\text {èmes }}$ Journées Nationales Génie Côtier - Génie Civil

La Rochelle, 29 au 31 mai 2018

DOI:10.5150/jngcgc.2018.024 C Editions Paralia CFL

disponible en ligne - http://www.paralia.fr - available online

\title{
Réponse morphologique de la plage du Lido de Sète suite à l'installation d'un ouvrage atténuateur de houle
}

\section{Clément BOUVIER $^{1,2}$, Yann BALOUIN ${ }^{2}$, Bruno CASTELLE ${ }^{1}$}

1. CNRS, UMR EPOC, Université de Bordeaux, Allée Geoffroy Saint-Hilaire, CS 50023, 33615 Pessac Cedex, France. clement.bouvier@u-bordeaux.fr,bruno.castelle@u-bordeaux.fr

2. BRGM, Université de Montpellier, 1039, rue de Pinville 34000 Montpellier, France. c.bouvier@brgm.fr,y.balouin@brgm.fr

\section{Résumé :}

Plusieurs relevés topo-bathymétriques et un système de suivi vidéo sont exploités afin d'étudier la réponse morphologique de la plage du Lido de Sète (Golfe du Lion) suite à l'installation d'un ouvrage type atténuateur de houle. Les observations montrent que l'atténuateur de houle a profondément impacté la morphologie et la dynamique des barres d'avant côte et du trait de côte. Contrairement à ce qui est très souvent constaté le long des plages sans barre pré-littorale, la formation de tombolo n'a pas suivi l'installation de l'ouvrage. Un nouveau scénario est observé dans lequel l'élargissement de la plage résulte de son couplage avec la nouvelle forme de barre plus linéaire et orientée de manière parallèle à la structure. L'atténuateur de houle impacte également la dynamique de l'avant côte dans le secteur adjacent situé à plusieurs kilomètres de la structure en affectant la variabilité spatiale du phénomène de Net Offshore Migration (NOM) des barres sableuses. Cette étude montre le rôle important joué par les barres sableuses dans la réponse du trait de côte suite à l'installation d'ouvrages maritimes.

\section{Mots-clés :}

Génie côtier, Ouvrage maritime, Barre d'avant côte, Trait de côte, Morphodynamique littorale.

\section{Introduction}

La gestion du trait de côte est aujourd'hui devenue un enjeu économique et sociétal de première importance. Alors que pendant des décennies le génie côtier s'est appuyé sur l'implantation d'ouvrages maritimes en dur (e.g. épis, brise-lames), il s'agit désormais d'envisager une gestion continue en intégrant la dynamique naturelle des environnements sableux ainsi que la mobilité du trait de côte. Des options dites "douces" sont donc apparues telles que les rechargements de plage ou bien l'installation d'ouvrages atténuateurs de houle.

Contrairement aux rechargements sableux, les ouvrages atténuateurs de houle ont reçu peu d'attention dans la littérature scientifique (RANASINGHE \& TURNER, 2006). Ces ouvrages jouent le rôle de récifs artificiels ou de barre sableuse naturelle en dissipant 


\section{Thème 2 - Dynamique sédimentaire}

l'énergie des vagues par déferlement bathymétrique. Ce type de structure présente de nombreux avantages (pas d'impact esthétique, forme et localisation relativement adaptable selon le besoin). Toutefois, les différents processus contrôlant le mode de réponse du trait de côte suite à la mise en place de ce genre d'ouvrage sont encore mal compris en particulier en présence de barre(s) pré-littorale(s).

\section{Site d'étude : le Lido de Sète}

Dans la région du Sud-Est de la France, l'étang de Thau est séparé de la Méditerranée par une étroite langue de sable relativement linéaire (figure 1a) exposée à de fortes pressions environnementales et économiques. La partie Nord du Lido définie ici comme le Lido de Sète est soumise à des taux d'érosion très élevés de l'ordre de $1 \mathrm{~m} / \mathrm{an}$ depuis une cinquantaine d'année (CERTAIN \& BARUSSEAU, 2005).

Afin de lutter contre l'érosion marine, un ouvrage atténuateur de houle a été installé début 2013 au niveau de la barre externe à $350 \mathrm{~m}$ du rivage (figure 1b). La structure en géotextile atteint $800 \mathrm{~m}$ de long, $12 \mathrm{~m}$ de large et sa crête se situe à environ 2 mètres de profondeur (figure 1c). Pour finaliser les travaux d'aménagement, un rechargement de $510000 \mathrm{~m}^{3}$ de sable a été réalisé au mois de Décembre 2014 derrière l'atténuateur de houle sur la plage émergée. Finalement, l'ouvrage a été rallongé pour atteindre $1 \mathrm{~km}$ de long en Octobre 2015.

\section{Données et méthode}

\subsection{Hydrodynamique}

Les conditions de vague sont enregistrées depuis le mois de Janvier 2011 toutes les 30 mn par un houlographe (bouée Candhis de la DREAL Occitanie) situé au large de Sète au niveau de l'isobathe $30 \mathrm{~m}$ (figure 1a). Les niveaux d'eau sont mesurés toutes les heures par un marégraphe (REFMAR) installé dans le port (figure 1a).

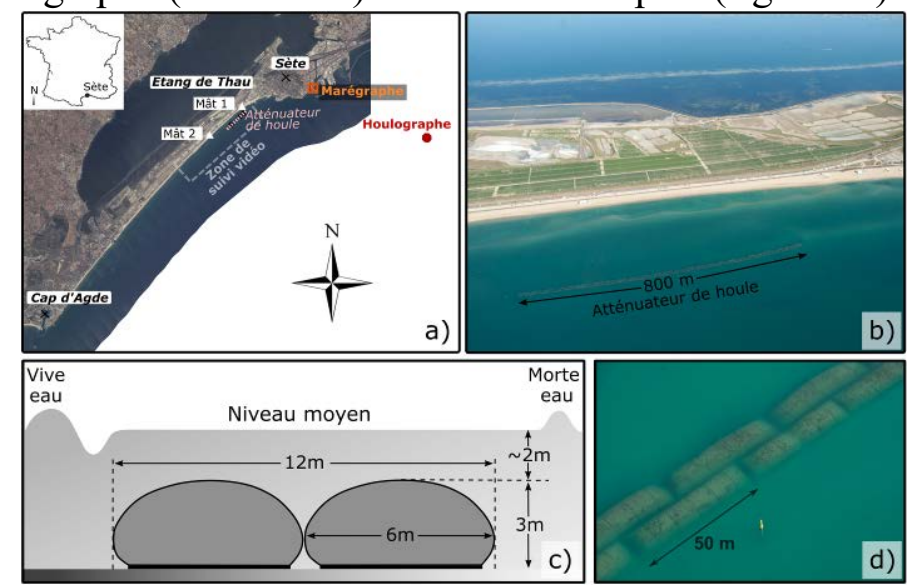

Figure 1. a) Site d'étude du Lido de Sète. $b, c$ et d) Dimensions et vue aérienne de l'atténuateur de houle (@) Thau Agglo). 


\section{XVèmes Journées Nationales Génie Côtier - Génie Civil \\ La Rochelle, 29 au 31 mai 2018}

\subsection{Levés topo-bathymétriques}

Le jeu de données topo-bathymétriques utilisé dans cette étude consiste en 6 levés réalisés de 2009 à 2016 avec des sondeurs monofaisceaux ou LiDAR. La précision verticale des levés par sondeur est de $0.10 \mathrm{~m}$, et celle des levés Lidar de $0.20 \mathrm{~m}$, pour une résolution horizontale autour de $5 \mathrm{~m}$ (ALEMAN et al., 2013). Les levés topobathymétriques sont interpolés (plus proche voisin) sur une grille régulière avec une résolution de $5 \mathrm{~m}$.

\section{1 $\underline{\text { Suivi vidéo }}$}

Un système de suivi vidéo a été mis en place depuis le mois d'avril 2011 et consiste en 8 caméras réparties sur 2 mâts de $20 \mathrm{~m}$ de haut et espacés de $2.5 \mathrm{~km}$ (figure 1a). Le système est configuré pour enregistrer chaque demi-heure des images instantanées et timex (moyennées sur $10 \mathrm{mn}$ ). Une fois géo-référencées, les images issues des caméras sont assemblées ce qui permet d'obtenir une zone de suivi continue (figure 2) sur une grille de $650 \mathrm{~m}$ de large (direction cross-shore; $\mathrm{X}_{\text {argus}}$ ) et $3350 \mathrm{~m}$ de long (direction longshore; $\left.\mathrm{Y}_{\text {argus}}\right)$.

La détection du trait de côte est réalisée en utilisant les images obliques sur lesquelles on détecte automatiquement par un critère teinte, saturation, luminosité (HSV) les zones humides des zones sèches (AARNINKHOF et al., 2003). Le trait de côte est extrait environ tous les 15 jours depuis le mois d'avril 2011 avec une erreur maximale sur sa position estimée à $5 \mathrm{~m}$ (BOUVIER et al., 2017).

La position des barres sableuses est déduite à partir des images timex en partant du principe que les zones de dissipation d'énergie (zones blanches) correspondent à la position des barres sableuses sous-jacentes qui font déferler les vagues. La position de la crête de barre est extraite avant et après chaque tempête de 2011 à 2016 avec une erreur maximale estimée à $12 \mathrm{~m}$ (BOUVIER et al., 2017) pour toute la période de suivi.

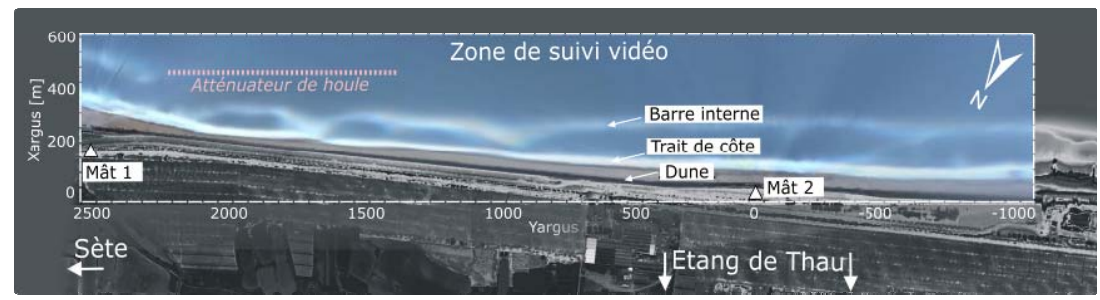

Figure 2. Image timex rectifiée du Lido de Sète.

La variabilité longitudinale de la barre, $\alpha_{\mathrm{Bar}}$ (ou du trait de côte, $\alpha_{\mathrm{Tdc}}$ ) est estimée en calculant la déviation standard entre la position cross-shore de la barre (ou du trait de côte) et la régression linéaire qui lui associée. L'obliquité de la barre $\left(\theta_{\mathrm{Bar}}\right)$ est définie 


\section{Thème 2 - Dynamique sédimentaire}

par son angle avec le pied de dune. Afin d'isoler la variabilité annuelle des analyses et d'en retirer les variations saisonnières, un filtre de Hamming (365 jours) est appliqué.

\section{Résultats}

\subsection{Réponse morphologique globale de la plage}

L'installation de l'atténuateur de houle en Janvier 2013 a eu un impact très important sur la morphologie des barres d'avant côtes (figure 3). Dans le secteur protégé (secteur $\mathrm{NE}$ ), les croissants réguliers au niveau de la barre interne ont totalement disparu pour laisser place à une géométrie de barre plus linéaire et orienté parallèlement à l'ouvrage. Dans le secteur adjacent (secteur SO), la barre interne a poursuivi sa migration vers le large, ce qui entraîna en Décembre 2013 la rupture du système de barre entre le secteur protégé par l'atténuateur de houle et le secteur adjacent (figure 3d). Pendant que la barre migrait vers le large dans le secteur SO, une nouvelle barre sableuse s'est progressivement formée à la côte en s'éloignant du rivage (figure $3 \mathrm{c}, \mathrm{d}$ et e) et s'est finalement rattachée à celle du secteur NE.

La structure fut initialement installée dans le secteur NE à $70 \mathrm{~m}$ au large de la crête de barre externe (figure $3 \mathrm{~g}$ ). Dans ce secteur, la barre externe initiale a disparu suite à la mise en place de la structure pour laisser place à une fosse de plus de $5 \mathrm{~m}$ de profondeur. Cet important volume de sédiment a migré vers le rivage pour venir engraisser progressivement le dos de la barre interne qui reste assez stable au cours du suivi jusqu'à un épisode de rechargement de plage en Décembre 2014. Au niveau du trait de côte, on observe une tendance érosive avant la mise en place de la structure. Cette tendance s'est inversée au cours de l'automne 2014 avec une plage qui a gagné environ $25 \mathrm{~m}$ de large en une seule année. Sur la côte adjacente, la migration vers le large de la barre interne fut accompagnée par le développement d'une nouvelle barre à la côte mais aussi d'un recul de $15 \mathrm{~m}$ du trait de côte entre Septembre 2011 et Novembre 2013 (figure 3h). 


\section{XVèmes Journées Nationales Génie Côtier - Génie Civil \\ La Rochelle, 29 au 31 mai 2018}
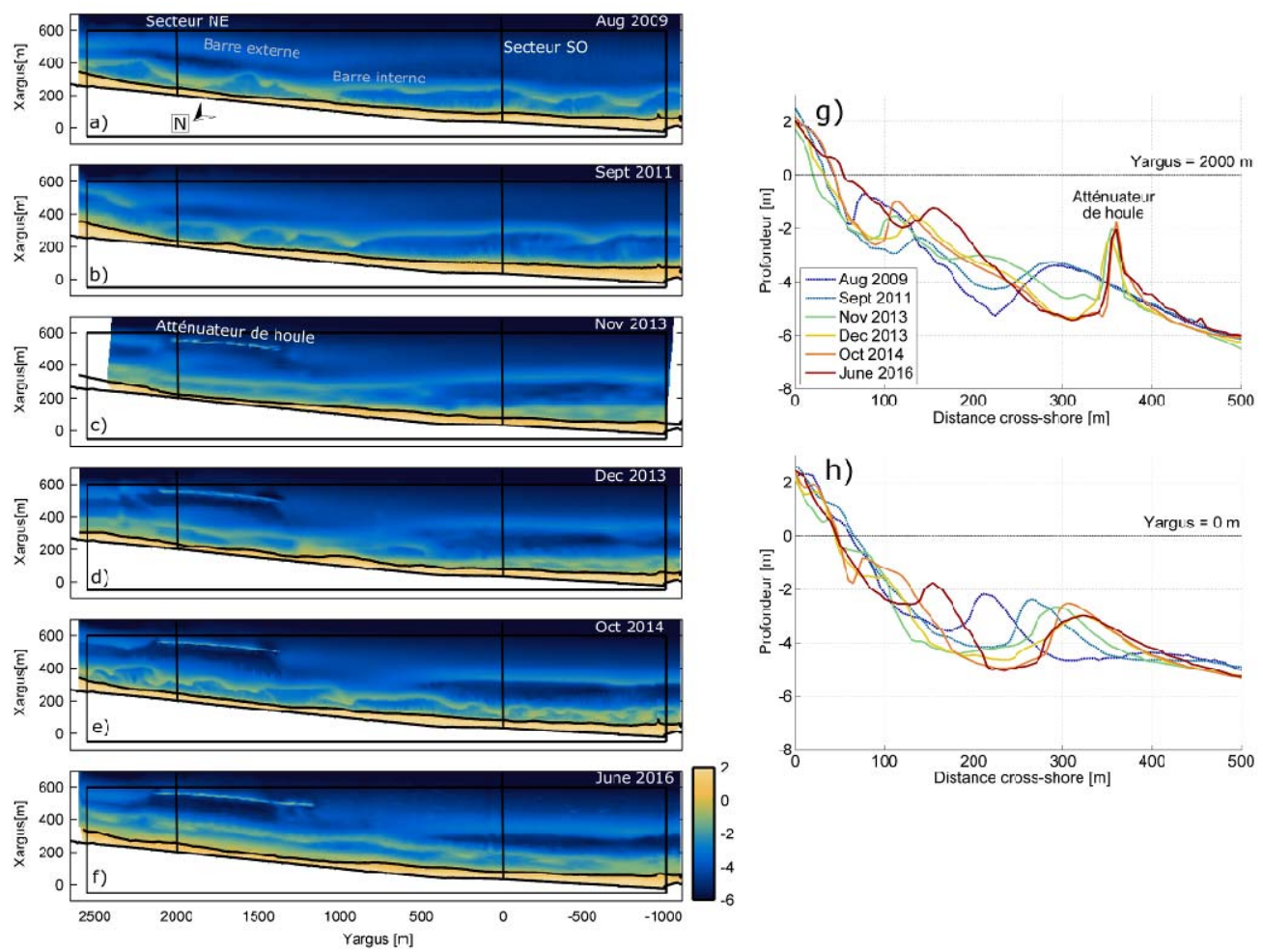

Figure 3. Vue en plan montrant les évolutions morphologiques de la plage ( $a$ à f).

Evolution des profils de plage g) dans le secteur protégé (NE) et h) adjacent (SO). Les positions des profils de plage sont illustrées par les traits noirs verticaux (a à f).

\subsection{Réponse à haute fréquence du système barre d'avant côte/trait de côte}

\section{Evolution cross-shore des barres sableuses}

La figure $4 \mathrm{~b}$ représente l'évolution temporelle des positions cross-shore moyennes des barres sableuses dans le secteur protégé et le secteur adjacent. Les conditions de vagues sont exprimées en terme de flux d'énergie (LONGUET-HIGGINS, 1970, cf. figure 4a). Les résultats montrent un changement de comportement important suite à l'installation de l'ouvrage. Initialement, la barre sableuse présente des évolutions similaires dans le secteur protégé et adjacent alors qu'elles se trouvent positionnées à des distances différentes de la côte (100 m contre $240 \mathrm{~m}$ respectivement).

Après la mise en place de l'ouvrage, les séries de coup de mer enregistrées en mars et décembre 2013 ont provoqué la migration vers le large de la barre d'avant côte. Cependant, la migration vers le large totale observée a été bien supérieure dans le secteur adjacent $(75 \mathrm{~m})$ que celle enregistrée dans le secteur protégé $(25 \mathrm{~m})$. La tempête de décembre 2013 a entrainé la rupture de la barre. Cette nouvelle configuration de l'avant côte a forcé le développement puis la migration vers le large d'une nouvelle barre dans le secteur adjacent (cycle de Net Offshore Migration, NOM) qui s'est progressivement alignée à la barre du secteur protégé par l'atténuateur. 


\section{Thème 2 - Dynamique sédimentaire}

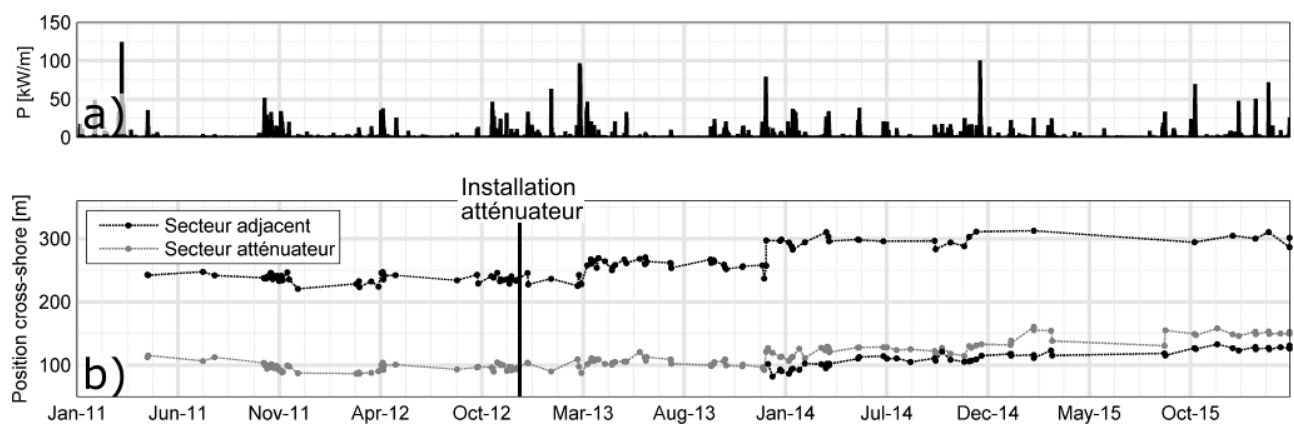

Figure 4. a) Energie des vagues au large. b) Position cross-shore des barres sableuses, moyennées sur $1 \mathrm{~km}$ dans le secteur protégé (gris) et adjacent (noir).

\section{Rotation et linéarisation du système de barre}

Dans le secteur protégé par l'ouvrage, l'évolution de la variabilité longitudinale de la barre et de son obliquité est respectivement illustrée par les figures $5 \mathrm{c}$ et d. Les conditions de vague $\left(\mathrm{H}_{\mathrm{s}}\right.$ et $\left.\mathrm{T}_{\mathrm{p}}\right)$ mesurées au large sont également fournies (figures $5 \mathrm{a}$ et b). Suite à la mise en place de l'atténuateur de houle, 3 phases principales peuvent être identifiées et sont illustrées sur les images timex (figure 6). La phase 1 correspond à la configuration de plage initiale avec une barre sableuse oblique $\left(\theta_{\mathrm{Bar}}=2^{\circ}\right.$, cf. figure $\left.5 \mathrm{~d}\right)$ et variable longitudinalement $\left(\alpha_{\mathrm{Bar}}=20 \mathrm{~m}\right.$, cf. figure $5 \mathrm{c}$ ). Les séries temporelles (figure $5 \mathrm{c}$ et d) montrent une décroissance de l'obliquité et de la variabilité longitudinale de la barre d'avant côte depuis la mise en place de l'atténuateur de houle en Janvier 2013. Cette décroissance se fait de manière tout à fait progressive à force d'endurer les coups de mer successifs. A la fin du suivi (phase 3, figure 6), la barre apparaît parallèle à la structure $\left(\theta_{\mathrm{Bar}}=-0.5^{\circ}\right)$ et bien plus linéaire $\left(\alpha_{\mathrm{Bar}}=5 \mathrm{~m}\right)$.

\section{Couplage du trait de côte}

Après mise en place de l'atténuateur de houle, on observe la décroissance progressive de la variabilité longitudinale du trait de côte (figure 5e). Une nouvelle forme de couplage semble avoir lieu entre la barre d'avant côte linéarisée et le trait de côte qui a adopté une nouvelle morphologie rectiligne à la fin du suivi (phase 3 ; figure 6). Cette évolution est étroitement reliée à l'élargissement local de la plage. Le trait de côte était initialement soumis à des vitesses de recul de $5 \mathrm{~m}$ par an (jusqu'à la phase 2). Corrélée avec la diminution de sa variabilité longitudinale, la plage a inversé sa tendance érosive et a gagné en moyenne $14 \mathrm{~m}$ en 1 an (figure 5f). 


\section{$X V^{\text {èmes }}$ Journées Nationales Génie Côtier - Génie Civil \\ La Rochelle, 29 au 31 mai 2018}

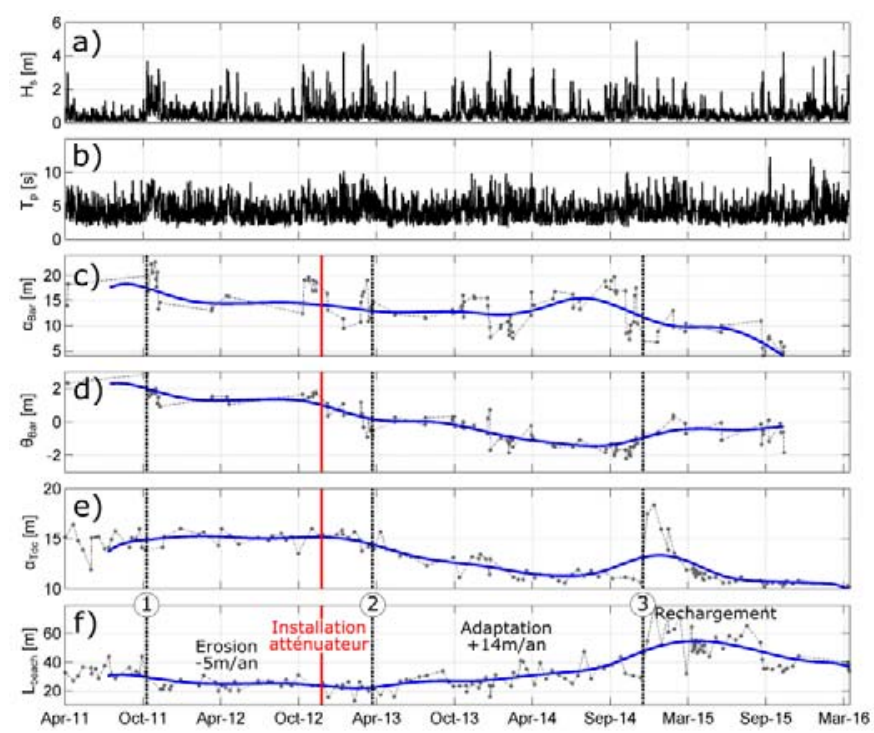

Figure 5. a) Hauteur et b) période des vagues mesurées au large. c) obliquité et d) variabilité longitudinale de la barre. e) variabilité longitudinale du trait de côte et f) largeur de plage moyenne. Les courbes bleues représentent le signal filtré avec une fenêtre de Hamming (365 jours).

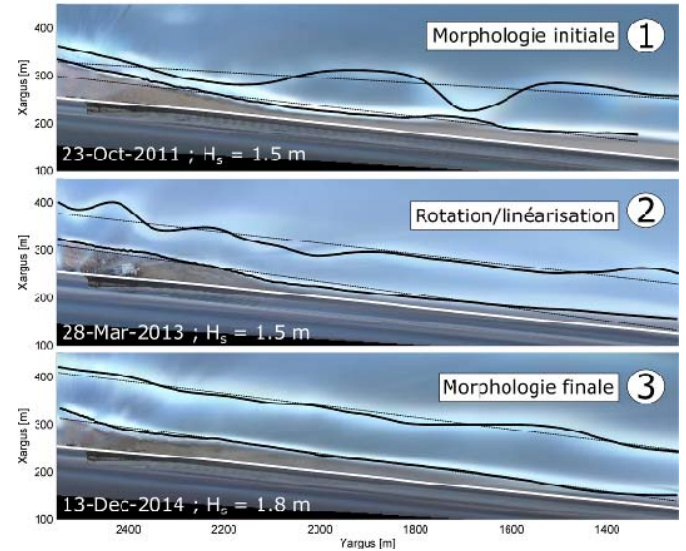

Figure 6. Image timex rectifiée montrant les 3 phases (indiquées figure 5) de la réponse du système barre/trait de côte suite à la mise en place de l'atténuateur de houle.

\section{Discussion et conclusion}

Les observations montrent que l'atténuateur de houle a profondément impacté la morphologie et la dynamique du système barre d'avant côte/trait de côte de la plage de Sète. Suite à l'installation de l'ouvrage, la barre se linéarise et s'oriente de manière parallèle à l'ouvrage. Alors que l'on aurait pu s'attendre à voir la variabilité longitudinale de la barre augmenter (due au déferlement supposé variable au-dessus de l'ouvrage), l'opposé fut observé avec une évolution vers un système plus linéaire.

L'atténuateur de houle semble également impacter la dynamique de l'avant côte dans le secteur adjacent situé à plusieurs kilomètres de la structure. Des cycles de migration de 


\section{Thème 2 - Dynamique sédimentaire}

barre d'avant côte vers le large ont déjà été observés à Sète (CERTAIN \& BARUSSEAU, 2005 ; ALEMAN et al., 2013). Depuis l'installation de l'ouvrage, les vitesses de migration obtenues dans le secteur protégé sont bien inférieures à celles observées dans le secteur adjacent, ce qui a provoqué la rupture de la barre. Cette dynamique est probablement reliée directement à l'atténuateur de houle qui, en dissipant l'énergie des vagues par un premier déferlement au large, favorise la stabilité de la barre interne dans le secteur protégé. Néanmoins, le mécanisme de rotation de barre, (fortement lié à la présence de l'ouvrage) a pu également favoriser le processus de rupture.

Contrairement à ce qui est souvent constaté, la formation de tombolo n'a pas suivi l'installation de l'atténuateur de houle. Un nouveau scénario est observé dans lequel l'élargissement de la plage dans le secteur protégé par l'ouvrage résulte de la linéarisation de la barre d'avant côte. Comme la barre devient linéaire, le trait de côte adopte une nouvelle morphologie rectiligne, ce qui provoque l'élargissement de la plage.

\section{Remerciements}

Le système vidéo utilisé dans ce travail est financé par Thau Agglo et le BRGM. Les données de vague et LiDAR ont été fournies par la DREAL-LR. CB remercie le BRGM, DREAL-Occitanie et Sète Agglo-Pôle Méditerranée pour leur soutien financier.

\section{Références bibliographiques}

AARNINKHOF S. G. J., TURNER I. L., DRONKERS T.D.T., CALJOUW M., NIPIUS M. (2003). A video technique for mapping intertidal beach bathymetry, Coastal Engineering, 49, 275-289. https://doi.org/10.1016/S0378-3839(03)00064-4

ALEMAN N., ROBIN N., CERTAIN R., BARUSSEAU J. P., GERVAIS M. (2013). Net offshore bar migration variability at a regional scale: inter-site comparison (Languedoc-Roussillon, France). J. Coast. Res. 2, 1715-1720 (Special Issue 65 International Coastal Symposium).

BOUVIER C., BALOUIN Y., CASTELLE B. (2017). Video monitoring of sandbarshoreline response to an offshore submerged structure at a microtidal beach. Geomorphology, 295, 297-305. https://doi.org/10.1016/..geomorph.2017.07.017

CERTAIN R., BARUSSEAU J.P. (2005). Conceptual modeling of sand bars morphodynamics for a microtidal beach (Sète, France). Bull. Soc. Geol. Fr. 176 (4), 343-354.

LONGUET-HIGGINS M. (1970). Longshore current generated by obliquely incident sea waves. J. Geophys. Res. 75, 6778-6801.

RANASINGHE R., TURNER I. L. (2006). Shoreline response to submerged structures: a review. Coast. Eng. 53, 65-79. https://doi.org/10.1016/j.coastaleng.2005.08.003 